ACM International Conference Proceeding SeriesVolume Part F134654, 20 December 2017, Pages 99-1039th International Conference on Education Technology and Computers, ICETC 2017; Barcelona; Spain; 20 December 2017 through 22 December 2017; Code 134654

\title{
Serious game for the virtual practice of the emplantillado in the constructive system of adobe with reinforced cane(Conference Paper)
}

- George, C.Email Author,

- Carlos, E.Email Author,

- David, M.Email Author

- View Correspondence (jump link)

- Peruvian University of Applied Sciences, Lima, Peru

\begin{abstract}
View references (9)
\end{abstract}
At present, the training of university students and the specialization of professionals are accompanied by a series of educational tools based on digital environments and applications for specific areas in education; In this process of application of the technology exists a methodology called Serious Game oriented towards some specific areas like Simulation in subjects of building and construction; Since this field is new in higher education, there are not many specific applications, such as the case of emplantillado for simulation of constructions using adobes reinforced with cane. The objective of this study is to demonstrate that the Serious game methodology, oriented to the teaching the course of the Preliminary Works of the Faculty of Architecture, can improve students learning and optimize their skills for the emplantillado in construction systems using the application for iPad. The application has been tested in a group of twelve students who were part of the experimental group in relation to the execution time, attempts made and qualification obtained; In contrast to evaluate the time to solve the test for emplantillado into de groups that performed the same exercise; an ANCOVA was used to evaluate the main effects and interactions to answer the null hypothesis. This ANCOVA model for balanced cases of type III includes a covariate that is correlated with the dependent variable and means on the dependent variables adjusted due the to the effect the covariate had on it. (C) 2017 Association for Computing Machinery.

Author keywords

Emplantillado of adobe and caneiPad AppSerious Game

Indexed keywords

Engineering controlled terms:

Compendex keywords
Education computingHand held computersPersonnel trainingReinforcementStuden

Building and constructionConstruction systemsConstructive systemsDependent var environmentEmplantillado of adobe and caneIpad appUniversity students 
Engineering main Serious games heading:

- ISBN: 978-145035435-6

- Source Type: Conference Proceeding

- Original language: English

- DOI: $10.1145 / 3175536.3175554$

- Document Type: Conference Paper

- Sponsors:

- Publisher: Association for Computing Machinery 